\title{
Preparation and Characterization of Microspheres of Albumin-Heparin Conjugates
}

\author{
GLEN S. KWON, * YOU HAN BAE, * SUNG WAN KIM,*,1 \\ HARRY CREMERS, $\dagger$ AND JAN FEIJEN $\dagger$ \\ * Department of Pharmaceutics and Center for Controlled Chemical Delivery, University of Utah, Salt Lake City, \\ Utah 84112; and $\dagger$ Department of Chemical Technology, University of Twente, Enschede, The Netherlands
}

Received August 21, 1990; accepted October 25, 1990

\begin{abstract}
Albumin-heparin microspheres have been prepared as a new drug carrier. A soluble albumin-heparin conjugate was synthesized by forming amide bonds between human serum albumin and heparin. After purification the albumin-heparin conjugate was crosslinked in a water-in-oil emulsion to form albuminheparin microspheres. The composition of the conjugate was determined by amino acid analysis. The swelling properties of albumin-heparin microspheres were investigated as a function of $\mathrm{pH}$ and ionic strength and compared with albumin microspheres. Albumin-heparin and albumin microspheres exhibited stimuli-sensitive swelling. Both microsphere systems exhibited low swelling at low $\mathrm{pH}$ and high swelling at higher $\mathrm{pH}$ caused by ionization of amino acids of serum albumin. The swelling of albumin-heparin microspheres was more sensitive toward ionic strength than that of albumin microspheres. This was due to the greater negative charge of the albumin-heparin microspheres. Surfaces of albumin-heparin and albumin microspheres were characterized by ESCA, contact angle measurements, electrophoresis, and scanning electron microscopy. Surface analysis indicated the presence of heparin at the albumin-heparin microsphere/water interface. @1991 Academic Press, Inc.
\end{abstract}

\section{INTRODUCTION}

In recent years, polymeric microspheres have been ubiquitously investigated as drug delivery systems (1). The interest in polymeric microspheres as drug carriers is due to the fact that these systems are injectable, can deliver drugs site specifically, and can release drugs in a controlled manner (2). The delivery of a drug, protected by a drug carrier or dosage form, to a specific site of action (e.g., tumor or specific organ ) may increase the therapeutic index of that drug (3).

In this contribution, the preparation of microspheres based on conjugates of serum albumin and heparin that could be used as a new drug delivery system is described. Cur-

\footnotetext{
${ }^{1}$ To whom correspondence should be addressed at Department of Pharmaceutics and Center for Controlled Chemical Delivery, University of Utah, 421 Wakara Way, Room 318, Salt Lake City, UT 84108.
}

rently there is much interest in the delivery of macromolecules, derived through biotechnology, due to their low oral bioavailability and short plasma half-lives (3). The viability of the microsphere approach has been investigated by Goosen et al. (4) with albumin microspheres for the sustained release of insulin. After subcutaneous injection of the insulinloaded microspheres, diabetic rats showed elevated blood insulin levels for up to 2 months. Immobilizing heparin in albumin microspheres may increase its blood and tissue compatibility. Many workers have attempted to inhibit thrombosis at polymer/blood interfaces by immobilizing heparin to polymer surfaces (5). Heparin is a highly negatively charged mucopolysaccharide and may enhance the hydrophilicity of albumin microspheres. Longo and Goldberg (6) have discussed the advantages of hydrophilic polymeric microspheres for drug delivery. A study 
on the release of Adriamycin from albuminheparin microspheres has been previously reported (7).

The composition of the albumin-heparin conjugate was determined by amino acid analysis. The size distribution of microspheres was measured. To establish the enhanced hydrophilicity of albumin-heparin microspheres the swelling as a function of $\mathrm{pH}$ and ionic strength of albumin-heparin microspheres in aqueous systems was compared to that of albumin microspheres.

The surface characteristics of albuminheparin and albumin microspheres were then examined. Surface properties largely determine the biocompatibility of microspheres when interfaced with body tissues and blood and they affect the distribution of intravenously administered microspheres through the process of opsonification (1). Due to their small size polymeric microspheres have a large specific surface area which would interface with the biological milieu. A thorough study of the surface characteristics of albumin-heparin microspheres was necessary to correlate in vivo performance with surface properties. The surfaces of albumin-heparin and albumin microspheres were therefore characterized by ESCA, contact angle measurements, electrophoresis, and scanning electron microscopy (SEM).

\section{MATERIALS AND METHODS}

\section{MATERIALS}

Human serum albumin (lyophilized and crystallized), olive oil, and 1-ethyl-3-(3-dimethylaminopropyl) carbodiimide (EDC) were obtained from Sigma (St. Louis, MO). Heparin (lot number LPP060143, average molecular weight 11,000), from porcine intestinal mucosa, was obtained from Diosynth (Oss, The Netherlands). Purified glutaraldehyde $(25 \%, \mathrm{w} / \mathrm{v})$ was purchased from Fisher (Pittsburgh, PA). Sodium cyanoborohydride was obtained from Aldrich (Milwaukee, WI). Blue Sepharose CL-6B and Diethylaminoethyl (DEAE)-Sepharose CL-6B were obtained from Pharmacia (Piscataway, NJ). Microconcavity slides were purchased from ClayAdams (Parsipanny, NJ). Pluronic F-68 was received from BASF (Parsipanny, NJ). Cellulose acetate (molecular weight cutoff 1000 and 3500 ) dialysis membranes and polytetrafluoroethylene membranes $(0.45-\mu \mathrm{m}$ pore size) were obtained from Gelman (Ann Arbor, MI). Sodium acetate $(0.1 \mathrm{~N})$ /acetonitrile buffer (HPLC grade) was obtained from VWR (Philadelphia, PA). Phenyl isothiocyanate was obtained from Pierce (Rockford, IL). Latexes ( 10 and $100 \mu \mathrm{m}$ ) were purchased from Coulter Counter (Hialeah, FL). All other chemicals were reagent grade.

\section{METHODS}

\section{Synthesis of Albumin-Heparin Conjugate}

The protocol used to synthesize the soluble albumin-heparin conjugate was based on the method of Hennink et al. (8). The procedure was slightly modified to inhibit denaturation of the serum albumin. A buffer was used to keep the $\mathrm{pH}$ constant during the course of the reaction without the addition of $\mathrm{HCl}$, and all steps were carried out at $4^{\circ} \mathrm{C}$. Heparin $(770$ $\mathrm{mg})$ and serum albumin $(2590 \mathrm{mg})$ were dissolved in $39.0 \mathrm{ml}$ phosphate buffer $(0.061 \mathrm{M}$ monopotassium phosphate, $5.33 \times 10^{-4} M$ disodium phosphate), $\mathrm{pH}$ 5.10. The solution was adjusted to $\mathrm{pH} 5.10$ with $0.10 \mathrm{M} \mathrm{HCl}$. Then, $32.5 \mathrm{mg}$ EDC was dissolved in $1.00 \mathrm{ml}$ distilled water and added to the heparin and serum albumin solution. This was repeated seven more times at 30 -min intervals. The total amount of EDC added was $260 \mathrm{mg}$. The $\mathrm{pH}$ was adjusted to $\mathrm{pH} 7.00$ with $1.00 \mathrm{M}$ $\mathrm{NaOH} 30$ min after the last addition of EDC. The resultant solution was then gently stirred for $20 \mathrm{~h}$. The solution was dialyzed (cellulose acetate, 1000 molecular weight cutoff) twice against $0.17 \mathrm{M} \mathrm{NaCl}, 0.025 M$ Tris $/ \mathrm{HCl}, \mathrm{pH}$ 7.00 , for $6 \mathrm{~h}$.

The albumin-heparin conjugate was purified from free serum albumin and heparin using a two-step liquid chromatography procedure. Heparin was removed by using a Blue 
Sepharose CL-6B column. The column was first equilibrated with two bed volumes of 0.17 $M \mathrm{NaCl}, 0.025 M$ Tris $/ \mathrm{HCl}, \mathrm{pH} 7.40$, buffer. A mixture of albumin-heparin conjugate and unbound heparin and serum albumin dissolved in $0.17 M \mathrm{NaCl}, 0.025 \mathrm{M}$ Tris $/ \mathrm{Hcl}, \mathrm{pH}$ 7.40 , buffer was applied to the column, and the heparin was eluted with one column volume of starting buffer. The albumin-heparin conjugate and serum albumin were eluted by a stepwise ionic strength gradient of $1.5 \mathrm{M}$ $\mathrm{NaCl}, 0.025 M$ Tris $/ \mathrm{HCl}, \mathrm{pH} 7.40$, buffer. Heparin was determined by using a toluidine blue assay described elsewhere (9). The fractions were assayed for serum albumin by measuring the absorbances at $280 \mathrm{~nm}$ with a UV spectrophotometer (UV-1, Pharmacia, Uppsala, Sweden). Fractions with absorbances greater than 0.5 were collected, pooled, and dialyzed (cellulose acetate, 3500 molecular weight cutoff) three times against 8 liters of deionized water. The sample was then freezedried (Lyph-Lock6, LabConCo, Kansas City, MO). The albumin-heparin conjugate and serum albumin were then redissolved in 0.30 $M \mathrm{NaCl}, 0.025 M$ Tris/ $\mathrm{HCl}, \mathrm{pH} 7.40$, buffer and applied to the DEAE-Sepharose CL-6B column which had been equilibrated with 0.30 $M \mathrm{NaCl}, 0.025 M$ Tris $/ \mathrm{HCl}$, pH 7.40, buffer. Serum albumin was eluted with one column of starting buffer. The albumin-heparin conjugate was eluted by a stepwise ionic strength gradient of $0.90 \mathrm{M} \mathrm{NaCl}, 0.025 M$ Tris $/ \mathrm{HCl}$, $\mathrm{pH}$ 7.40. The fractions of albumin-heparin conjugate that exhibited an absorbance greater than 0.5 were collected, pooled, and dialyzed against 8 liters of deionized water. The conjugate was then freeze-dried. All separation and dialysis procedures were carried out at $4^{\circ} \mathrm{C}$.

\section{Preparation of Albumin-Heparin and Albumin Microspheres}

Microspheres were synthesized using a protocol similar to that of Burger et al. (10). A $125-\mathrm{ml}$ volume of olive oil was placed in a baffled cell ( 10 ) and stirred at a predetermined rate for $30 \mathrm{~min}$. The $100 \mathrm{mg}$ of albumin-heparin conjugate or serum albumin, which had been dissolved in $400 \mu 1$ of distilled water at $4^{\circ} \mathrm{C}$, was injected dropwise into stirred olive oil at $25^{\circ} \mathrm{C}$, and stirring was continued for 15 min. A predetermined amount of purified glutaraldehyde $(25 \%, \mathrm{w} / \mathrm{v})$ to make 1 to $4 \%$ (w/v) of the added albumin-heparin solution was then added along with twice the equimolar amount of sodium cyanoborohydride solution. The crosslinking reaction was allowed to proceed for $3.5 \mathrm{~h}$. A $100-\mathrm{ml}$ aliquot of glycine $(10 \%, \mathrm{w} / \mathrm{v})$ was then added to quench unreacted aldehyde groups and allowed to proceed for $30 \mathrm{~min}$. Subsequently $60 \mathrm{ml}$ of acetone was added and the emulsion stirred for $1 \mathrm{~min}$. The microspheres were isolated by centrifugation (MSE, VWR, Philadelphia, $\mathrm{PA}$ ) at $1000 \mathrm{rpm}$ for $15 \mathrm{~min}$. The supernatant was then decanted. The microspheres were resuspended in acetone, collected on a $0.45-\mu \mathrm{m}$ polytetrafluoroethylene membrane, and washed and dehydrated with acetone. The collected microspheres were suspended in acetone, sieved between appropriate sized $\mathrm{mi}$ crosieves (U.S. Standard, Newark Wire Cloth, Newark, NJ), and washed with isotonic phosphate buffer (PBS) $\left(2.00 \times 10^{-3} \mathrm{M}\right.$ potassium phosphate, $8.00 \times 10^{-3} M$ disodium phosphate, $0.145 \mathrm{M} \mathrm{NaCl}$ ), pH 7.40, and distilled water. The microspheres were air-dried for 24 $\mathrm{h}$, vacuum-dried for an additional $24 \mathrm{~h}$, and stored frozen. Albumin-heparin and albumin gels used for contact angle measurements were prepared by in situ crosslinking of the proteins in syringes at $4^{\circ} \mathrm{C}$.

\section{Composition}

The composition of the albumin-heparin conjugate was determined by amino acid analysis. Briefly, the method first involved hydrolysis of the sample. The determinations of serum albumin and heparin were carried out independently because hydrolysis conditions for proteins are too harsh for polysaccharides and result in degradation (11). Hydrolysis of serum albumin was carried out with $6 \mathrm{~N} \mathrm{HCl}$ 
at $106^{\circ} \mathrm{C}$ for $20 \mathrm{~h}$. For the analysis of heparin, hydrolysis was done with $4 \mathrm{~N} \mathrm{HCl}$ at $100^{\circ} \mathrm{C}$ for $16 \mathrm{~h}$. Subsequently the amino acids and glucosamine were derivatized using phenyl isothiocyanate to form phenylthiocarbamoyl (PTC) derivatives. The PTC derivatives were then separated using sodium acetate $(0.1 \mathrm{~N}) /$ acetonitrile buffer as the mobile phase with reverse phase column (Ultrasphere OPS, 4.6 $\mathrm{mm} \times 15 \mathrm{~cm}$, Waters, San Francisco, CA) using HPLC (1084B, Waters, San Francisco, CA). The amount of PTC derivative was determined by measuring absorbance at $254 \mathrm{~nm}$ with a UV spectrophotometer (484, Waters). Heparin standards were analyzed by the same procedure.

\section{Analysis of Size Distribution}

The size distribution of swollen and unswollen albumin-heparin and albumin microspheres $(n=300)$ was determined by phase-contrast light microscopy (Biophot, Nikon, Tokyo, Japan ) using a calibrated graticule. Calibration was done with latexes of known size.

\section{Swelling}

Equilibrium swelling of microspheres was studied as a function of $\mathrm{pH}$ and ionic strength. To examine the effects of $\mathrm{pH}$ on the swelling of albumin-heparin and albumin microspheres, a universal buffer, Britton and Robinson (12), was used and the ionic strength of the buffer was adjusted to a constant value of 0.21 with sodium chloride. The effect of ionic strength on swelling was examined using an isotonic PBS, $\mathrm{pH} 7.40$, with sodium chloride added to adjust the ionic strength of the media. The swelling was also studied as a function of glutaraldehyde used for crosslinking. Equilibrium swelling was determined in isotonic PBS, $\mathrm{pH}$ 7.40. All swelling experiments were done at $25^{\circ} \mathrm{C}$. The swelling ratio

$$
q=\frac{V_{\text {swollen }}}{V_{\text {dried }}}
$$

was determined by measuring the diameter of albumin-heparin and albumin microspheres ( $n=50$ ) by phase-contrast light microscopy (Biophot, Nikon) and by assuming a spherical geometry. Albumin-heparin and albumin microspheres sieved between 38 and $53 \mu \mathrm{m}$ with an average dry diameter of $40 \pm 5 \mu \mathrm{m}$ were used for these studies. Standard error of the means (s.e.m.) of the swelling ratio was calculated by propagating the error of the microsphere diameter (13).

\section{Surface Characterization of Albumin- Heparin and Albumin Microspheres}

ESCA analysis. The chemical nature of albumin-heparin and albumin microsphere surfaces was studied by ESCA (5950B, Hewlett-Packard, Palo Alto, CA). The X-ray source was a monochromatic $\mathrm{AlK} \alpha_{1,2}$ and had an energy of $1487 \mathrm{eV}$ and $400 \mathrm{~W}$ power at the anode. To inhibit charging, an electron flood gun bathed the surface of the microspheres at $6.0 \mathrm{eV}$. Albumin-heparin and albumin microspheres were prepared by vacuum-drying. Albumin-heparin microspheres were also prepared by freeze-drying. Samples were frozen at $-60^{\circ} \mathrm{C}$ with dry ice in acetone and then freeze-dried (Lyph-Lock6, LabConCo). Atomic percentages of each sample were calculated by utilizing area sensitivity techniques (14).

Contact angle measurements. The contact angles of albumin-heparin and albumin gels were determined using a goniometer (A-100, Rame-Hart, Inc., Mountain Lakes, NJ). Droplets of double-distilled water were carefully placed via a syringe onto the surface of the gel. The advancing contact angle was determined by measuring the angle of the tangent to the drop with the surface at the gel/water/ air meeting point. Measurements were carried out at $25^{\circ} \mathrm{C}$ in triplicate and standard deviations were calculated. The water contents of the gels were determined by measuring the weight of the gels swollen in deionized water and the weight of the dried gels. The water contents (in percent) were defined as the 
weight of the swollen gel minus the weight of the dried gel divided by the weight of the swollen gel.

Electrophoresis measurements. The zeta potentials of albumin-heparin and albumin microspheres were measured by electrophoresis (3.0, Zetameter Inc., Long Island City, NY). The average diameter of the microspheres was $60 \pm 27 \mu \mathrm{m}$. An isotonic PBS, 7.40 , was used for the determination of zeta potentials, and all experiments were done at $25^{\circ} \mathrm{C}$. The zeta potential of albumin microspheres was determined using $0.1 \%$ Pluronic F-68, a nonionic surfactant, to prevent flocculation. The Smoluchowski equation was used to calculate the zeta potentials,

$$
\zeta=\frac{\mu \eta}{\epsilon \epsilon_{0}}
$$

(assuming $\kappa R>100$ ), where $\mu$ is defined as the electrophoretic mobility. This value is experimentally obtained by determining the velocity of microspheres in an applied electric field by light microscopy. Measurements were carried out in triplicate and standard deviations were calculated. $\eta$ is the viscosity of the bulk medium. $\epsilon \epsilon_{0}$ is the dielectric permittivity of the medium. $\kappa$, the reciprocal of the Debye length, is determined by the composition of the medium

$$
\kappa=\left(\frac{e^{2}}{\epsilon \epsilon_{0} k T} \sum c_{\mathrm{i}} z_{\mathrm{i}}^{2}\right)^{1 / 2} .
$$

$e$ is the elementary charge, $k$ is the Boltzmann constant, $T$ is the absolute temperature, and $c_{\mathrm{i}}$ and $z_{\mathrm{i}}$ are the concentration and valency of the ionic species. $R$ is the radius of albuminheparin microspheres. The Debye length is less than $10 \AA$ under isotonic conditions.

$S E M$. The surfaces of albumin-heparin and albumin microspheres were studied using SEM. Microspheres were vacuum-dried overnight and then placed on stubs and sputtercoated with $\mathrm{Au} / \mathrm{Pd}$ using a sputter coater (Hammer III, Technics, Alexandria, VA). The surfaces were examined with SEM (JSM-35, JEOL, Peabody, MA).

\section{RESULTS AND DISCUSSION}

To immobilize heparin within an albumin matrix, soluble albumin-heparin conjugate was first synthesized. Since heparin and serum albumin do not interact at physiological conditions ( 15), heparin was covalently bound to serum albumin via amide bonds. The carboxyl groups of serum albumin and heparin are preactivated by EDC in the presence of an external nucleophile, the lysine residue, and thus the formation of the $\mathrm{N}$-acylurea is inhibited (16). By using a buffer the $\mathrm{pH}$ did not have to be adjusted during the course of the reaction. The $\mathrm{pH}$ did slightly increase with each addition of EDC as hydrogen ions were consumed in the reaction (16). The $\mathrm{pH} 30 \mathrm{~min}$ after the last addition of EDC was 5.24.

Purification of the albumin-heparin conjugate was accomplished with liquid chromatography, and the elution profiles are shown in Figs. 1 and 2. Heparin was isocratically eluted from the Cibacron Blue F3G-A column. When a stepwise ionic strength gradient was applied a skewed peak was observed. This peak represents the serum albumin and the albumin-heparin conjugate. The skewed peak is due to the difference in affinity between the serum albumin and albumin-heparin conjugate toward the immobilized dye. It was not possible to resolve the two components on this column. Serum albumin was eluted isocratically on the DEAE-Sepharose CL-6B column, and using a stepwise ionic strength gradient the albumin-heparin conjugate was eluted from the column (Fig. 2). The greater affinity of the albumin-heparin conjugate toward DEAE-Sepharose CL-6B was a result of the greater negative charge associated with the albumin-heparin conjugate relative to serum albumin. The percentage yield of the albuminheparin conjugate after purification was $30 \%$ based on the initial weight of the serum albumin and heparin.

Albumin-heparin microspheres were prepared by crosslinking the conjugate in a waterin-oil emulsion. The percentage yield of albumin-heparin and albumin microspheres

Joumal of Colloid and Interface Science, Vol. 143, No. 2, May 1991 


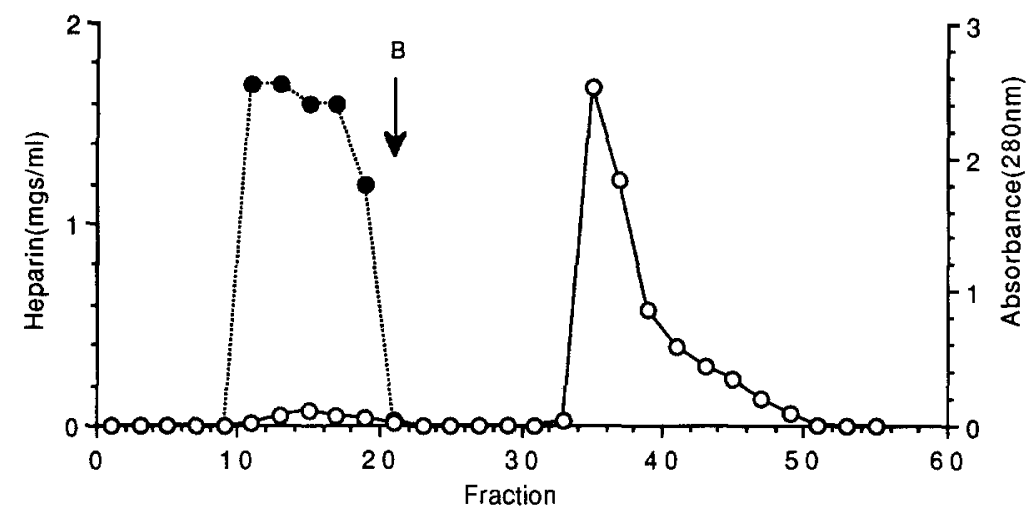

FIG. 1. Elution profile of heparin, serum albumin, and albumin-heparin conjugate on Blue Sepharose CL-6B. Initial buffer: $0.17 M \mathrm{NaCl}, 0.025 M$ Tris/HCl, pH 7.40; B: $1.50 M \mathrm{NaCl}, 0.025 M$ Tris/HCl, $\mathrm{pH}$ 7.40. Heparin, closed circles; albumin-heparin conjugate and serum albumin, open circles.

was $90 \%$. Dried microspheres are free-flowing light tan powders. Albumin-heparin microspheres, crosslinked with glutaraldehyde, which was not reduced, formed imine bonds which may undergo subsequent reactions and are brownish in appearance. Imine bonds are unstable in aqueous media and thus should undergo reduction (17).

The size of the microspheres may be controlled by a number of experimental variables: oil phase viscosity, oil phase volume, protein concentration, aqueous phase volume, and stirring rate $(10)$. All variables were kept constant and the size was altered by varying the stirring rate and by sieving. The fate of microspheres after intravenous injection has been shown to be largely dependent on microsphere size and has been exploited for the site-specific delivery of antineoplastics $(1,18)$. Albuminheparin microspheres that were made using a stirring rate of $500 \mathrm{rpm}$ had an average diameter of $60 \pm 27 \mu \mathrm{m}$ in the swollen form. Microspheres were prepared with diameters ranging from 1 to $300 \mu \mathrm{m}$.

The composition of the albumin-heparin conjugate is summarized in Table 1 . The content of serum albumin was directly quantified by obtaining the number of moles of amino

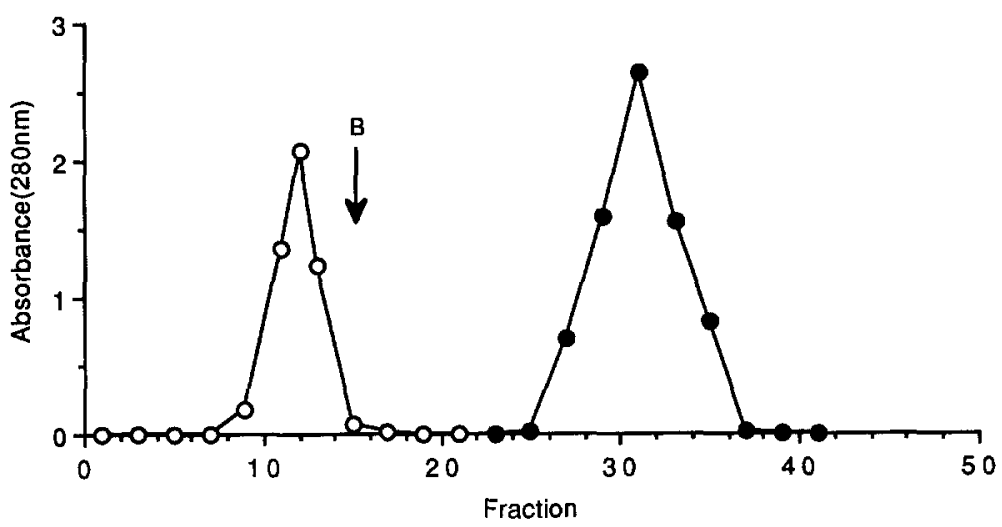

FIG. 2. Elution profile of serum albumin and albumin-heparin conjugate on DEAE Sepharose CL-6B. Initial buffer: $0.30 \mathrm{M} \mathrm{NaCl}, 0.025 \mathrm{M}$ Tris $/ \mathrm{HCl}, \mathrm{pH} 7.40 ; \mathrm{B}: 0.90 \mathrm{M} \mathrm{NaCl}, 0.025 \mathrm{M}$ Tris $/ \mathrm{HCl}, \mathrm{pH} 7.40$. Serum albumin, open circles; albumin-heparin conjugate, closed circles. 
TABLE I

Composition of Albumin-Heparin Conjugate

\begin{tabular}{ccc}
\hline $\begin{array}{c}\text { Heparin content } \\
{[\mathrm{wt} \%]}\end{array}$ & $\begin{array}{c}\text { Albumin content } \\
\text { [wt \%] }\end{array}$ & $\begin{array}{c}\text { Albumin/heparin } \\
{[\mathrm{mol} / \mathrm{mol}]}\end{array}$ \\
\hline $9.54 \pm 0.4^{b}$ & $90.5 \pm 0.3^{b}$ & $1.58 \pm 0.07^{b}$ \\
\hline
\end{tabular}

${ }^{a}$ Determination by amino acid analysis.

${ }^{b} \mathrm{SD}(n=3)$.

acids. The heparin content was determined by quantifying the number of moles of glucosamine. The weight percentage of heparin in the albumin-heparin conjugate was found to be $9.54 \%$ by amino acid analysis. The amount of incorporated heparin is quite large relative to other similar heparin-immobilized hydrogel systems which contain $0.64 \%$ by weight heparin (19). The heparin is expected to be bioactive, and a systematic in vitro study of the blood compatibility of albumin-heparin microspheres is currently in progress.

Figure 3 shows that $\mathrm{pH}$ can act as an external stimulus to modulate the swelling of albumin-heparin microspheres; the degree of swelling is dependent on the crosslink density. A hydrogel network which has a higher crosslink density exhibits a greater retractive force

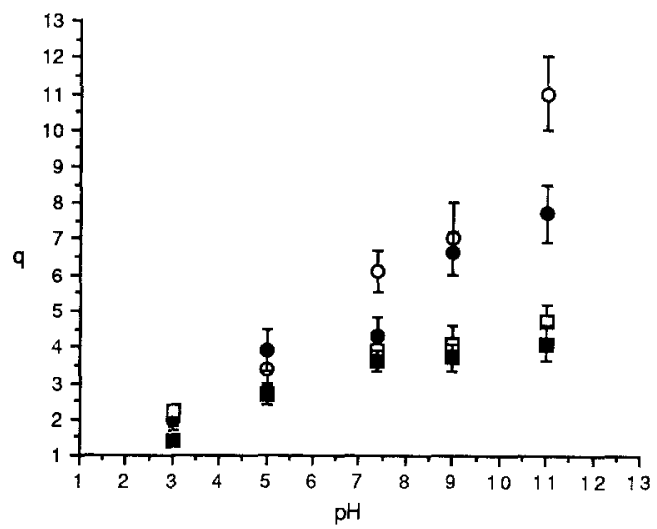

FIG. 3. pH-dependent swelling of albumin-heparin microspheres with varying crosslinking $(\%, \mathrm{w} / \mathrm{v}$, glutaraldehyde). Ionic strength, $0.21 ; 25^{\circ} \mathrm{C} .1 \%$, open circles; $2 \%$, closed circles; $3 \%$, open squares; $4 \%$, closed squares. S.E.M. $(n=25)$. which prevents the gel from expanding (20). The swelling changes reflect the ionization of functional groups of amino acids (e.g., Asp, Glu, and Lys) of serum albumin and carboxyl groups of heparin which is dependent on $\mathrm{pH}$. An increased number of like negative charges associated with the polymer chains result in expansion of the chain which is opposed by polymer network retractive forces and polymer-polymer interactions $(20,21)$. In Fig. 4, the $\mathrm{pH}$-dependent swelling of albumin-heparin and albumin microspheres at $1 \%(\mathrm{w} / \mathrm{v})$ glutaraldehyde is shown. The swelling of albumin microspheres is sensitive toward $\mathrm{pH}$ and also reflects the ionization of amino acids. Counterions in solution may effectively neutralize the repulsion of like charges along the polymer chain through electrostatic interaction, thus shielding the charge on the polymer chain and decreasing swelling (Fig. 5). The swelling of albumin-heparin microspheres is greater and exhibits more sensitivity toward ionic strength than albumin microspheres due to the higher negative charge of albumin-heparin microspheres. The swelling of synthetic hydrogels may be altered by the degree of crosslinking, and Fig. 6 illustrates that this is also possible with albumin-heparin microspheres. Stimuli-sensitive swelling with syn-

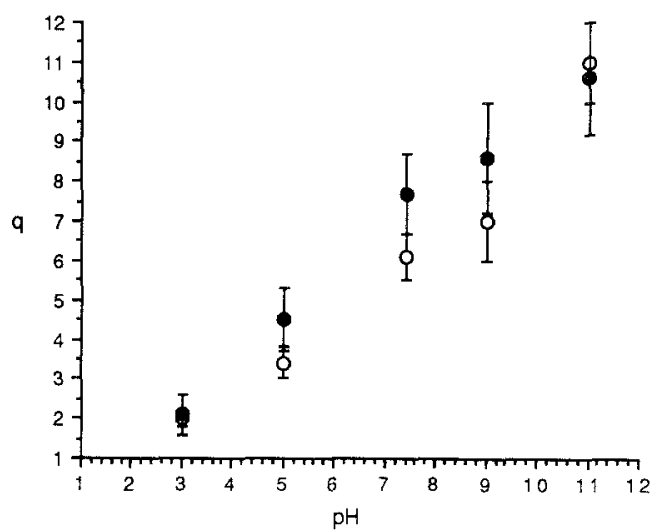

FIG. 4. pH-dependent swelling of albumin-heparin and albumin microspheres $(1 \%, \mathrm{w} / \mathrm{v}$, glutaraldehyde). Ionic strength, $0.21 ; 25^{\circ} \mathrm{C}$. Albumin-heparin microspheres, open circles; albumin microspheres, closed circles. S.E.M. $(n=25)$. 


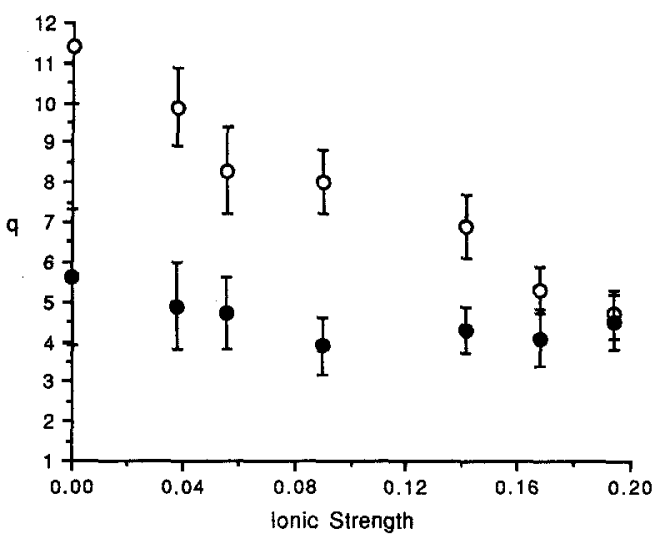

FIG. 5. Albumin-heparin and albumin microspheres $(1 \%, w / v$, glutaraldehyde $)$. Swelling dependence on ionic strength, pH $7.40,25^{\circ} \mathrm{C}$. Albumin-heparin microspheres, open circles; albumin microspheres, closed circles. S.E.M. $(n=25)$.

thetic hydrogels has been successfully used to load drugs into the matrix after hydrogel preparation (22). This process may now be utilized with albumin-heparin microspheres.

Previous investigators have shown that serum albumin may adsorb at the water/oil interface in the emulsion during the preparation of the albumin microspheres and that serum albumin undergoes a change in conformation so that hydrophobic regions of the protein are preferentially exposed to the oil

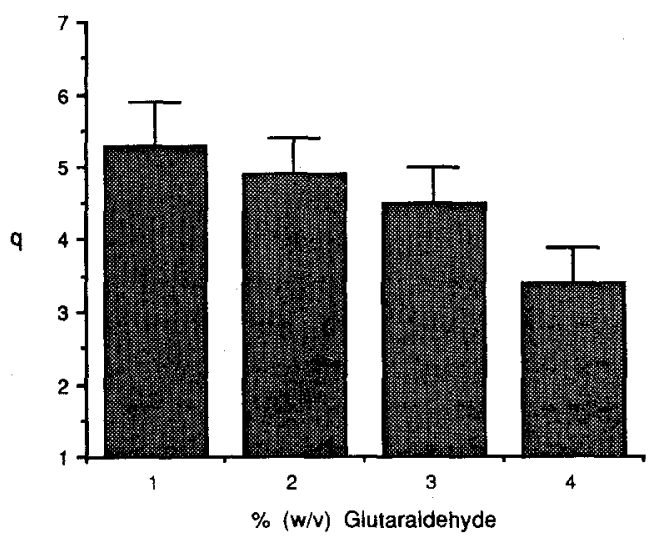

FIG. 6. Albumin-heparin microspheres' swelling in isotonic PBS, pH $7.40,25^{\circ} \mathrm{C}$. Dependence on degree of crosslinking. phase (23). The formation of an adsorbed layer composed of denatured serum albumin results in marginally stable microspheres without heat or chemical crosslinking. The preferential adsorption of serum albumin, in the case of the albumin-heparin conjugate, may result in the heparin moiety being directed away from the surface of the microsphere. The properties of the albumin-heparin microsphere surface would then be determined by serum albumin. In order to ascertain the presence of heparin at the surface of the albumin-heparin microsphere the surfaces were examined using ESCA. The results of the ESCA studies are summarized in Table II. Albumin microspheres exhibit a preponderance of carbon, relative to oxygen, at the surface. The elemental composition of albumin-heparin microsphere surfaces was shown to be dependent on the method of microsphere drying prior to ESCA analysis. Albumin-heparin microspheres that had been vacuum-dried display relative abundances of carbon, oxygen, and nitrogen similar to those of albumin microspheres, suggesting that the surface contains largely serum albumin. When albumin-hep-

TABLE II

ESCA Data for Albumin-Heparin and Albumin Microspheres

\begin{tabular}{|c|c|c|c|}
\hline Sample & Element & $\begin{array}{l}\text { Binding } \\
\text { energy } \\
(\mathrm{eV})\end{array}$ & Atom $\%$ \\
\hline \multirow[t]{4}{*}{ Albumin microspheres } & $\mathrm{C}(1 s)$ & 282 & 71.6 \\
\hline & $\mathrm{N}(1 s)$ & 396 & 8.9 \\
\hline & $O(1 s)$ & 528 & 17.4 \\
\hline & $\mathrm{Na}(2 s)$ & 61 & 1.7 \\
\hline \multirow{4}{*}{$\begin{array}{l}\text { Albumin-heparin } \\
\text { microspheres }\end{array}$} & $\mathrm{C}(1 s)$ & 282 & 74.3 \\
\hline & $\mathrm{N}(1 s)$ & 396 & 6.28 \\
\hline & $\mathrm{O}(1 s)$ & 528 & 16.2 \\
\hline & $\mathrm{Na}(2 s)$ & 61 & 3.17 \\
\hline \multirow{5}{*}{$\begin{array}{l}\text { Albumin-heparin } \\
\text { microspheres }\end{array}$} & $\mathrm{C}(1 s)$ & 282 & 63.8 \\
\hline & $\mathrm{N}(1 s)$ & 396 & 8.1 \\
\hline & $O(1 s)$ & 528 & 19.9 \\
\hline & $S(2 p)$ & 168 & 0.6 \\
\hline & $\mathrm{Na}(2 s)$ & 61 & 2.4 \\
\hline
\end{tabular}

\footnotetext{
${ }^{a}$ Vaccum-dried

${ }^{b}$ Freeze-dried.
} 
arin microspheres are freeze-dried ESCA analysis shows that the surface now contains sulfur and shows a decrease in the carbon:oxygen ratio which is consistent with the presence of heparin at the surface. These results imply that heparin is present at the surface of microspheres when interfaced with aqueous systems. When the albumin-heparin microspheres are in contact with air the surfaces may undergo reorientation so that serum albumin is largely at the surface. Such reorientation processes at polymer/water and polymer/air interfaces are well documented and occur to minimize interfacial free energies (24). Heparin at the surface is more likely to exhibit bioactivity relative to heparin in the interior of the microsphere.

Surface wettability was determined by contact angle measurements. Contact angles of albumin-heparin and albumin gels and water contents of the gels are summarized in Table III. Both gels exhibit high water contents. The low contact angles are consistent with the hydrated nature of the gels. The albumin-heparin gel has a slightly lower contact angle possibly due to the immobilized heparin at the surface. Contact angles are measures of gel/ water interfacial free energies. King et al. have demonstrated that the interfacial free energy at the gel/water interface approaches zero as the water content of the gels approaches $30 \%$ (25). From these results, it may be surmised that the interfacial free energy approaches zero for both albumin-heparin and albumin gels.

Albumin-heparin and albumin microspheres both have a net negative surface charge (Table IV). Zeta potential approximates surface potential; for albumin-heparin micro-

\section{TABLE III}

Contact Angle Measurements and Percentage Water Contents of Albumin-Heparin and Albumin Hydrogels

\begin{tabular}{lcc}
\hline \multicolumn{1}{c}{ Hydrogel } & $\theta_{\text {(vater) }}$ & $\% \mathrm{H}_{2} \mathrm{O}$ \\
\hline Albumin-heparin & $10 \pm 2^{a}$ & 96 \\
Albumin & $15 \pm 3^{a}$ & 68 \\
\hline
\end{tabular}

${ }^{a} \mathrm{SD}(n=3)$.
TABLE IV

Zeta Potentials of Albumin-Heparin and Albumin Microspheres

\begin{tabular}{cc}
\hline \multicolumn{1}{c}{ Microsphere } & Zeta potential (mV) \\
\hline Albumin-heparin $^{a}$ & $-40.0 \pm 4^{c}$ \\
Albumin $^{b}$ & $-17.0 \pm 2^{c}$ \\
\hline
\end{tabular}

${ }^{a}$ Suspension medium, isotonic PBS, pH 7.40.

${ }^{b}$ Suspension medium, 0.1\% (w/v) Pluronic F-68, PBS, pH 7.40 .

${ }^{c} \mathrm{SD}(n=3)$.

spheres the zeta potential was $-40.0 \mathrm{mV}$. The highly negatively charged nature of the surface accounts for its colloidal stability in isotonic phosphate buffer, $\mathrm{pH} 7.40$, without the presence of surfactants and is consistent with the presence of heparin at the surface. The negative surface charge may result in a decreased interaction with biosurfaces since most of the surfaces also have a net negative charge (26). Albumin microspheres were found to have a zeta potential of $-17.0 \mathrm{mV}$ which is in good agreement with the literature (10). Generally, polymeric microspheres require surfactants for colloidal stability; this may cause deleterious effects on drug release and affect performance in vivo. Surfactants may possibly be toxic and affect tissue interactions.

SEM micrographs (Figs. 7 and 8 ) taken at a magnification of $40 \times$ and $400 \times$ of albuminheparin microspheres suggest that they have a homogeneous structure without macropores (27). The porosity of homogeneous hydrogels has been described to be related to the void volume between individual polymer chains (27). Drug release from homogeneous hydrogels is expected to be slower compared to macroporous systems. Dry albumin-heparin microspheres appear spherical, have a smooth surface, and appear similar to albumin microspheres.

\section{SUMMARY}

In conclusion, the preparation of albuminheparin microspheres has been described. It was shown that the albumin-heparin and al- 

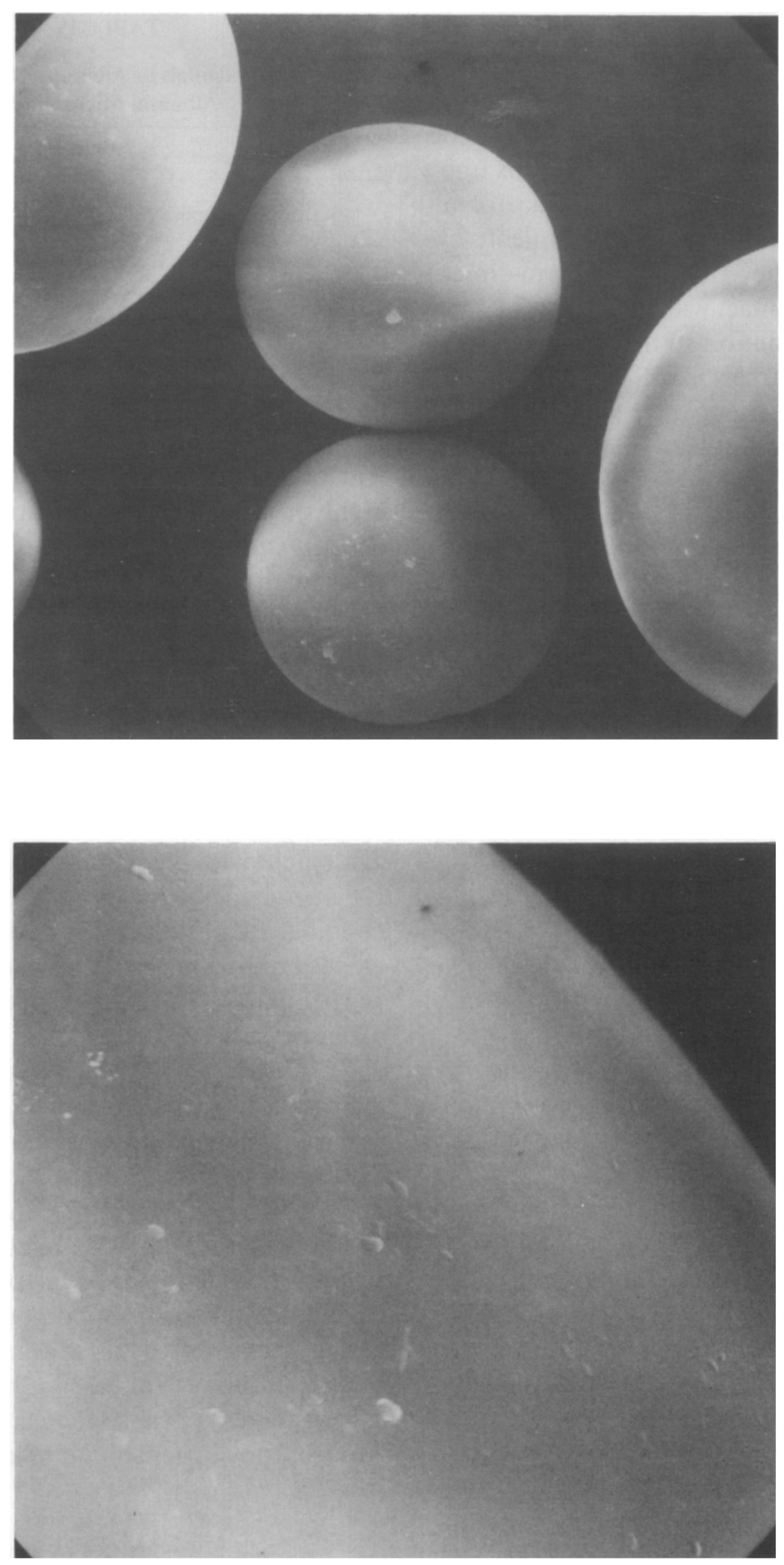

FIG. 7. SEM micrographs of albumin-heparin microspheres. Magnification: 40×, 400×. 

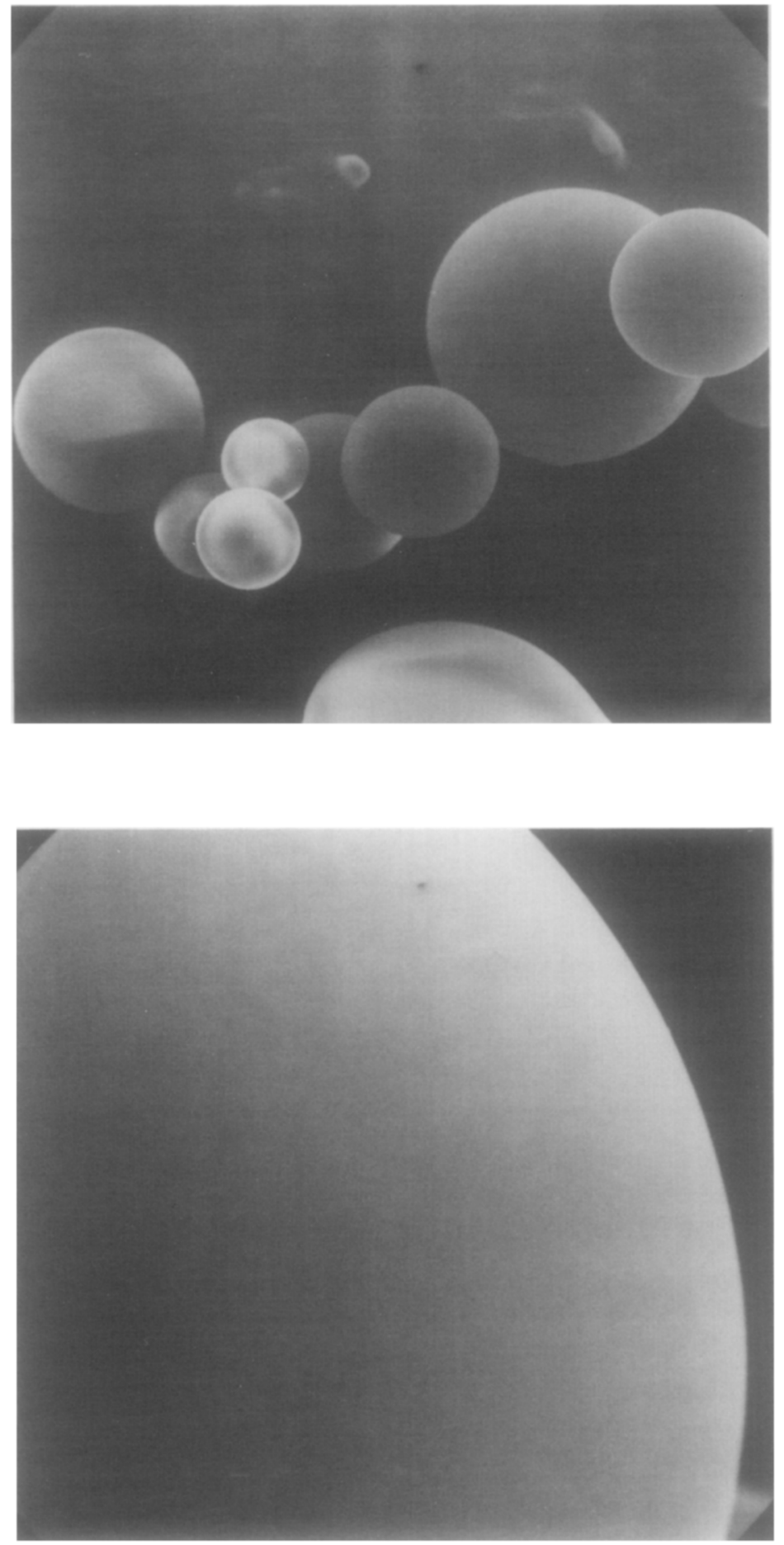

FIG. 8. SEM micrographs of albumin microspheres. Magnification: $40 \times, 400 \times$. 
bumin microspheres are stimuli-sensitive hydrogels characterized by a high degree of swelling. Albumin-heparin microspheres exhibited an enhanced hydrophilicity and an increased negative charge relative to albumin microspheres due to the immobilized heparin. ESCA studies indicated that heparin is present at the surface of the albumin-heparin microsphere when interfaced with an aqueous system, but the surface may undergo reorientation when interfaced with air, showing a preponderance of serum albumin. Both surfaces are quite wettable as evidenced by contact angle measurements. The surface of albuminheparin microspheres was more negatively charged than that of albumin microsphere surfaces which resulted in greater colloidal stability precluding the need for the addition of surfactants for stabilization. In vitro biodegradation, drug loading, and release of macromolecules from albumin-heparin microspheres are currently being investigated.

\section{ACKNOWLEDGMENT}

This work was supported, in part, by TheraTech, Inc., Salt Lake City, Utah.

\section{REFERENCES}

1. Lynn, L., Davis, S. S., Illum, L., and Davis, M. C., Biomed. Polym 4, 1 (1988).

2. Tomlinson, E., J. Controlled Release 2, 385 (1985).

3. Tomlinson, E., Adv. Drug Delivery Rev. 1, 87 (1987).

4. Goosen, M., Leung, Y., Chou, S., and Sun, A. M., Biomatter. Med. Devices Artif. Organs 10, 205 (1982).

5. Ebert, C. D., and Kim, S. W., in "Medical Applications of Controlled Release" (R. S. Langer and D. L. Wise, Eds.), p. 77. CRC Press, Boca Raton, FL, 1984.

6. Longo, W., and Goldberg, E., in "Methods in Enzymology" (Kenneth J. Widder and Ralph Green, Eds.), vol. 112, p. 18. Academic Press, New York, 1985.

7. Cremers, H. F. M., Feijen, J., Kwon, G., Bae, Y. H., Kim, S. W., Noteborn, H. P., and McVie, J. G., J. Controlled Release 11, 167 (1990).

8. Hennink, W., Feijen, J., Ebert, C. D., and Kim, S. W., Thromb. Res. 29, 1 (1983).
9. Smith, P. K., Mallia, A. K., and Harmanson, G., Anal. Biochem. 109, 466 (1980).

10. Burger, J. J., Tomlinson, E., Mulder, E., and McVie, J. G., Int. J. Pharm. 23, 333 (1985).

11. Neuberger, A., and Marshall, R. D., in "Glycoproteins: Their Composition, Structure and Function" (A. Gottschalk, Ed.), p. 190. Elsevier, Amsterdam, 1966.

12. Dawson, R. M., "Data for Biochemical Research," p. 474. Oxford Univ. Press, London/New York, 1969.

13. Peters, D. G., Hayes, J. M., and Hieftje, G. M., "Chemical Separations and Measurements," p. 19. Saunders, Philadelphia, 1974.

14. Andrade, J. D., in "Surface and Interfacial Aspects of Biomedical Polymers" (J. D. Andrade, Ed.), p. 105. Plenum, New York, 1984.

15. McKay, E. J., and Laurell, C. B., J. Lab. Clin. Med. 95, 69 (1980).

16. Rich, D. H., and Singh, J., in "The Peptides: Analysis, Synthesis, and Biology" (E. Gross and J. Meienhofer, Eds.), p. 242. Academic Press, New York, 1979.

17. Sokoloski, T. D., and Royer, G., in "Microspheres and Drug Therapy: Pharmaceutical, Immunological and Medical Aspects" (S. S. Davis, L. lllum, J. G. McVie, and E. Tomlinson, Eds.), p. 295. Elsevier, Amsterdam, 1984.

18. Gupta, P. K., Hung, C. T., and Perrier, D. G., Int. J Pharm. 33, 137 (1986).

19. Rollason, G., and Sefton, M., Thromb. Res. 44, 517 (1986).

20. Flory, P. J., "Principles of Polymer Chemistry," p. 584. Cornell Univ, Press, New York, 1954.

21. Seigel, R., and Firestone, B. A., Macromolecules 21, 3254 (1988).

22. Hoffman, A. S., Afrassiabi, A., and Dong, L. C., $J$. Controlled Release 4, 213 (1986).

23. Widder, K., Flouret, G., and Senyei, A., J. Pharm. Sci. 68, 79 (1979).

24. Andrade, J. D., and Chen, W. Y., Surf. Interface Anal. 8, 253 (1986).

25. King, R. N., Andrade, J. D., Ma, S. M., Gregonis, D. E., and Brostrom, L. R., J. Colloid Interface Sci. 103, 62 (1985).

26. Norde, W., in "Microspheres and Drug Therapy: Pharmaceutical, Immunological and Medical Aspects" (S. S. Davis, L. Illum, J. G. McVie, and E. Tomlinson, Eds.), p. 43. Elsevier, Amsterdam, 1984.

27. Ratner, B. D., in "Biocompatibility of Clinical Implant Material" (D. F. Williams, Ed.), p. 145. CRC Press, Boca Raton, FL, 1981 